\title{
A radiomics nomogram for the prediction of overall survival in patients with hepatocellular carcinoma after hepatectomy
}

Qinqin Liu ${ }^{1,2,3}$, Jing Li ${ }^{2}$, Fei Liư ${ }^{1}$ Weilin Yang ${ }^{4}$, Jingjing Ding ${ }^{5}$, Weixia Chen ${ }^{4}$, Yonggang Wei ${ }^{1}$, Bo Li ${ }^{*}$ and Lu Zheng ${ }^{2^{*}}$

\begin{abstract}
Background: Hepatocellular carcinoma (HCC) is associated with a dismal prognosis, and prediction of the prognosis of HCC can assist in therapeutic decision-makings. An increasing number of studies have shown that the texture parameters of images can reflect the heterogeneity of tumors, and may have the potential to predict the prognosis of patients with HCC after surgical resection. The aim of this study was to investigate the prognostic value of computed tomography $(\mathrm{CT})$ texture parameters in patients with HCC after hepatectomy and to develop a radiomics nomogram by combining clinicopathological factors and the radiomics signature.

Methods: In all, 544 eligible patients were enrolled in this retrospective study and were randomly divided into the training cohort $(n=381)$ and the validation cohort $(n=163)$. The tumor regions of interest (ROIs) were delineated, and the corresponding texture parameters were extracted. The texture parameters were selected by using the least absolute shrinkage and selection operator (LASSO) Cox model in the training cohort, and a radiomics signature was established. Then, the radiomics signature was further validated as an independent risk factor for overall survival (OS). The radiomics nomogram was established based on the Cox regression model. The concordance index (Cindex), calibration plot and decision curve analysis (DCA) were used to evaluate the performance of the radiomics nomogram.

(Continued on next page)
\end{abstract}

\footnotetext{
* Correspondence: hxcdlibo@126.com; xqyyzl1@163.com

'Department of Liver Surgery, Center of Liver Transplantation, West China Hospital, Sichuan University, 37 Guo Xue Road, Chengdu 610041, Sichuan Province, China

${ }^{2}$ Department of Hepatobiliary Surgery, the Second Affiliated Hospital of Army

Medical University, No. 183 Xinqiao High Street, Shapingba District,

Chongqing 400037, China

Full list of author information is available at the end of the article
}

(c) The Author(s). 2020 Open Access This article is licensed under a Creative Commons Attribution 4.0 International License, which permits use, sharing, adaptation, distribution and reproduction in any medium or format, as long as you give appropriate credit to the original author(s) and the source, provide a link to the Creative Commons licence, and indicate if changes were made. The images or other third party material in this article are included in the article's Creative Commons licence, unless indicated otherwise in a credit line to the material. If material is not included in the article's Creative Commons licence and your intended use is not permitted by statutory regulation or exceeds the permitted use, you will need to obtain permission directly from the copyright holder. To view a copy of this licence, visit http://creativecommons.org/licenses/by/4.0/. The Creative Commons Public Domain Dedication waiver (http://creativecommons.org/publicdomain/zero/1.0/) applies to the data made available in this article, unless otherwise stated in a credit line to the data. 
(Continued from previous page)

Results: The radiomics signature was formulated based on 7 OS-related texture parameters, which were selected in the training cohort. In addition, the radiomics nomogram was developed based on the following five variables: afetoprotein (AFP), platelet-to-lymphocyte ratio (PLR), largest tumor size, microvascular invasion (MVI) and radiomics score (Rad-score). The nomogram displayed good accuracy in predicting OS (C-index $=0.747$ ) in the training cohort and was confirmed in the validation cohort (C-index $=0.777)$. The calibration plots also showed excellent agreement between the actual and predicted survival probabilities. The DCA indicated that the radiomics nomogram showed better clinical utility than the clinicopathologic nomogram.

Conclusion: The radiomics signature is a potential prognostic biomarker of HCC after hepatectomy. The radiomics nomogram that integrated the radiomics signature can provide a more accurate estimation of OS than the clinicopathologic nomogram for HCC patients after hepatectomy.

Keywords: CT texture analysis, Hepatocellular carcinoma, Overall survival, Prediction, Nomogram

\section{Background}

HCC is the fifth most common malignancy and ranks as the third most common cause of cancer-related death worldwide $[1,2]$. Surgical resection is the preferred treatment option for individuals with HCC [3]. However, the long-term prognosis of patients with hepatocellular carcinoma after resection is dismal, as the 5-year survival rate is only $25-55 \%$ and the 5 -year recurrence rate is 60 $100 \%$ [4-7]. The prognosis of HCC is influenced by numerous factors, and thus, early prediction of the prognosis is of great significance for the long-term management and effective treatment of patients with this disease. Currently, the Barcelona Clinic Liver Cancer (BCLC) system is the most recognized staging system for HCC worldwide and is a widely used tool that guides prognostic prediction and treatment decisions [3]. Despite this, the BCLC classification is still controversial and has limited predictive power [8-10]. Therefore, it is worthwhile to explore additional reliable and pragmatic methods that can be used to evaluate the prognosis of HCC.

Previous imaging studies were based on the shape, density and enhancement of tumors [11], which did not quantify the information of the images and were easily affected by the subjectivity of the radiologists. It is known that malignant tumors are composed of heterogeneous cells and their surrounding microenvironment, and that intra-tumor heterogeneity is associated with tumor angiogenesis and biological behavior, which can be assessed through imaging traits. Radiomics is an emerging field, in which high-dimensional information can be extracted from medical images. Texture analysis (TA), an image post-processing technique, can be used to evaluate the potential heterogeneity of lesions based on a large set of quantitative features [12-14]. Emerging studies have shown that texture features have the potential to differentiate tumor types, monitor therapeutic response, identify regional lymph node metastasis of malignant tumors and predict prognosis $[15,16]$. Huang et al. [17] developed a radiomics nomogram, which exhibited favorable accuracy for the preoperative prediction of lymph node metastasis in patients with colorectal cancer. Wu et al. [18] developed a radiomics nomogram for the preoperative prediction of lymph node metastasis in bladder cancer and found that CT texture parameters were independent predictors of response to chemotherapy. Ahn et al. [19] demonstrated that lower skewness in the $2 \mathrm{D}$ analysis and a narrower SD in the $3 \mathrm{D}$ analysis were useful predictors of chemotherapeutic response in patients with colon cancer liver metastasis. In addition, the radiomics signature could be used to predict preoperative individualized MVI status and early recurrence of HCC [20, 21]. Furthermore, previous studies have shown that radiomics features are correlated with gene expression, gene mutations and epigenetic alterations through capturing the tumor phenotypes, which are associated with underlying gene expression patterns of cancer and may reflect cellular proliferation, invasion, metastasis and drug resistance of the tumors [22-26]. Segal et al. [23] reported that variations in 116 gene modules can be reconstructed from 28 imaging traits. However, due to the limited number of studies, additional studies are needed to confirm the potential association between radiophenotype and gene expression $[27,28]$. In the future, accurate and quantitative imaging information based on an artificial intelligence automatic image recognition and diagnosis system in combination with clinical data can help doctors evaluate patient survival. It plays an important role in clinical decision making, treatment planning and postoperative long-term follow-up, and provides new opportunities for individual precise treatment.

The underlying correlation among radiomics features, pathology and survival is not clear, and relatively few studies have addressed the efficacy of TA in prognostic prediction. The intratumor heterogeneity can reflect the biological characteristics of the tumor, which may be of prognostic significance. The purpose of this study is to 
explore the prognostic value of preoperative CT texture parameters for patients who underwent radical hepatectomy. In addition, a prognostic nomogram is proposed on the basis of texture parameters to provide useful references for precision medicine.

\section{Methods}

\section{Patients}

In all, 544 consecutive patients with $\mathrm{HCC}$ who underwent hepatectomy in the Department of Liver Surgery at West China Hospital between January 2013 and December 2016 were enrolled according to the following inclusion criteria: (1) patients who underwent initial radical hepatectomy with pathologically confirmed HCC; (2) Child-Pugh A or B liver function; (3) no preoperative treatments such as radiofrequency ablation, transcatheter arterial chemoembolization (TACE) and chemotherapy; and (4) preoperative contrast-enhanced CT performed within 4 weeks. The exclusion criteria were as follows: (1) CT images with invisible lesions or severe artifacts; (2) patients with benign or mixed types of liver tumor; (3) those who underwent simultaneous hepatectomy and radiofrequency ablation; (4) liver transplantation performed during the course of disease; (5) incomplete clinical or follow-up data. We randomly divided the eligible patients at a ratio of 7:3 into 2 groups: the training cohort $(n=381)$ and the validation cohort $(n=163)$. This study was approved by the Committee of Ethics of West China Hospital of Sichuan University. The clinicopathologic variables were collected, including patient demographics, laboratory data, tumor characteristics, surgical outcomes, and postoperative pathological data.

\section{Patient follow-up and surveillance}

All patients were followed-up by telephone or outpatient visit during the first month after surgery and then every 3 months thereafter until November 2019. The routine examinations, which included serum AFP levels, routine blood tests, serum biochemistry, hepatitis B virus deoxyribonucleic acid (HBV-DNA), abdominal ultrasonography and contrast-enhanced CT/MRI, were performed at each outpatient follow-up visit. OS was calculated as the period from the time of surgery to the time of either death or last follow-up.

\section{Image acquisition and imaging texture analysis}

All CT scans of the liver were acquired on a Siemens scanner (Siemens Somatom Definition FLASH, Siemens Healthcare, Erlangen, Germany). After $6 \mathrm{~h}$ of fasting, patients received an intravenous administration of $1.5 \mathrm{ml} /$ $\mathrm{kg}$ of Iohexol (Jiangsu Yangtze River Pharmaceutical Group Co., Ltd., Taizhou, China; $300 \mathrm{mg}$ of iodine/ml) at a rate of $2-3 \mathrm{ml} / \mathrm{s}$. Then, images acquisition was performed in the arterial phase and venous phase at 25 and $70 \mathrm{~s}$, respectively. The following scan parameters were used: tube voltage of $120-140 \mathrm{kV}$, tube current of $210 \mathrm{~mA}$, pitch of 4.0 , matrix size of $512 \times 512$, slice thickness of $5 \mathrm{~mm}$, and a high spatial resolution algorithm.

The target images were retrieved from the Picture Archiving and Communication System in Digital Imaging and Communications in Medicine (DICOM) format and transferred to Mazda software (version 4.6) for further TA. All manual segmentations were performed by an abdominal radiologist with 5 years of experience and were verified by a senior radiologist with 20 years of experience. The two-dimensional regions of interest (ROIs) delineated the largest cross-sectional area of the tumors on the preoperative portal venous phase images. For patients with multifocal HCCs, the ROIs of the largest tumor were selected for further analysis. The CT images on the portal venous phase were used for radiomics feature extraction because hypovascular HCC may influence the accuracy and reproducibility of the ROIs delineation on the arterial phase [29], and the previous studies showed the excellent predictive performance of texture features in this phase [30,31].

According to the segmented tumors, 270 texture features that reflected tumor heterogeneity were extracted, including the following 5 categories: (1) histogram features; (2) co-occurrence matrix; (3) run-length matrix; (4) autoregressive model; (5) wavelet transform [32]. Detailed information on the texture features is available in Supplementary Table S1.

\section{Feature selection and radiomics signature building}

We used the LASSO Cox regression model to select the features that were most associated with the survival status of the training cohort, and a 10-fold cross validation was used to reduce overfitting [33]. LASSO is a data analysis method that can shrink the coefficients of variables unrelated to survival to zero, and thus, the features with non-zero coefficient were selected [34]. The optimal tuning parameter was determined by minimum criteria (minimum lambda). The radiomics signature was built via a linear combination of selected features multiplied by their corresponding non-zero coefficients. Then, the Rad-score was calculated for each patient.

\section{Model construction and evaluation}

The patients were stratified into either the high-risk or low-risk groups according to the threshold of the Radscore calculated by ROC curve analysis. The difference between the survival curves of the high-risk and low-risk groups was assessed in the training cohort and then validated in the validation cohort. Univariate and multivariate Cox regression analyses were performed in the 
training cohort to determine the potential independent risk factors. Then a radiomics nomogram that integrates the radiomics signature and the independent clinicopathological risk factors according to the result of the multivariate analysis was constructed to predict postoperative survival status. The discrimination ability of the nomogram was evaluated using the $\mathrm{C}$-index. The calibration performance was measured by the calibration curve, which described the agreement between the predicted and observed survival probability. The clinical value of the nomogram was assessed in the whole cohort by DCA [35], which was generated by calculating the net benefits at different threshold probabilities.

\section{Statistical analysis}

The statistical analysis was performed with SPSS version 22.0 software (Chicago, IL, USA) and R software (version 3.5.1; http://www.R-project.org). Continuous variables were presented as the mean \pm standard deviation for normally distributed variables or as the median (interquartile range) for non-normally distributed variables. Differences between the two groups were compared using the $t$-test or Mann-Whitney $U$ test. Additionaly, categorical variables were expressed by frequency (percentage) and assessed by Pearson's chi-square test or Fisher's exact test. ROC curve analysis was used to determine the optimal cutoff values based on the maximum Youden index. Survival curves were calculated using the Kaplan-Meier method and were compared using the log-rank test. The Cox regression analysis was used for both univariate and multivariate analyses. Variables with $P$-values $<0.10$ in the univariable analysis were introduced into the multivariate Cox proportional hazards model to further determine the independent prognostic factors with a backward stepwise selection. The LASSO Cox regression model analysis was based on the glmnet package. The nomogram and calibration curve were established using the rms package, while the DCA was performed using the dca. $\mathrm{R}$ package. The predictive performance of the nomograms was evaluated by the C-index and was compared using the Rcorrp.cens package in Hmisc in R. A $P$ value $<0.05$ was considered statistically significant.

\section{Results}

\section{Patient demographics and clinicopathological characteristics}

In all, 544 patients who met the inclusion criteria were retrospectively analyzed. The comparison of the clinicopathological characteristics between the training cohort $(n=381)$ and the validation cohort $(n=163)$ is shown in Table 1. The median follow-up time was 28.8 months (range, 15.1-40.5 months) in the training cohort and 27.2 months (range, 16.9-39.5 months) in the validation cohort. No significant differences were observed in the baseline characteristics between the two groups $(P>$ $0.05)$, which suggests similarity between the cohorts.

\section{Construction and validation of the radiomics signature}

We evaluated the ROIs of hepatic tumors from preoperative CT images and extracted a total of 270 texture features. Then, the LASSO Cox regression model was used to select the most significant features for survival prediction (Fig. 1). When the minimum lambda was 0.034 , seven potential predictors of OS-related features with non-zero coefficients were screened out in the training cohort. A radiomics signature was constructed with the selected features and their respective weights. The Rad-score for each patient can be calculated using the following formula: $\operatorname{Rad}$-score $=S(0,1)$ Correlat "0.026 + S (0,3) Correlat"0.036 + Horzl_GLevNonU*0.312 + 45dgr_RLNonUni*0.024 + 45dgr_GLevNon U*0.036 + Sigma* $(-0.068)+$ WavEnLH_s-4*0.037). According to the optimum cut-off Rad-score based on the maximum Youden index in the training cohort, all patients were classified into the high-risk group (Rad-score $\geq-$ 0.559 ) or the low-risk group (Rad-score $<-0.559$ ). The OS was compared between the two groups using a KaplanMeier analysis (Fig. 2) in both the training and validation cohorts. The 1, 3 and 5-year OS rates of the low-risk group were $91.7,82.1$ and $78.7 \%$, respectively, which were significantly higher than those of the high-risk group in the training cohort $(71.0,45.5$ and $35.5 \%, P<0.001)$. The performance of the radiomics signature was confirmed in the validation cohort, and a significant difference was observed in the 1, 3 and 5-year OS rates between the high-risk and low-risk groups $(72.3,40.9,36.8 \%$ vs. $93.8,83.4,81.0 \%, P<$ 0.001 ). We also observed that patients with lower Radscores generally had a better OS.

To further evaluate the association between the radiomics signature and the clinicopathological features, the clinicopathological data of the high-risk and low-risk groups were compared (Table 2). In the training cohort, no significant difference was found between the low-risk and high-risk groups with regard to age, sex, BMI, HBsAg, HBV-DNA, liver cirrhosis, Child-Pugh classification, previous abdominal surgery, comorbidities, CEA, CA19-9, TBIL, DBIL, ALT, Albumin, NLR, ASA grade, tumor number, hepatectomy, and differentiation. However, the high-risk group was positively associated with higher AFP $(P=0.007)$, higher AST $(P<0.001)$, higher PLR $(P=0.001)$, larger tumor size $(P<0.001)$, more hemorrhage $(P<0.001)$, higher incidence of intraoperative transfusion $(P=0.019)$, the presence of MVI $(P=0.003)$, an incomplete tumor capsule $(P<0.001)$ and higher Rad-score $(P<0.001)$.

Development and validation of the radiomics nomogram The results of the univariate analysis based on the training cohort are displayed in Table 3. According to the 
Table 1 Clinicopathological factors of 544 patients who underwent radical hepatectomy

\begin{tabular}{|c|c|c|c|}
\hline Variables & Training cohort $(n=381)$ & Validation cohort $(n=163)$ & $P$ \\
\hline Age, years & $51.3 \pm 11.2$ & $50.2 \pm 11.5$ & 0.301 \\
\hline Sex & & & 0.410 \\
\hline Male & $324(85.0 \%)$ & $143(87.7 \%)$ & \\
\hline Female & $57(15.0 \%)$ & $20(12.3 \%)$ & \\
\hline $\mathrm{BMI}, \mathrm{Kg} / \mathrm{m}^{2}$ & & & 0.713 \\
\hline$<18.5$ & $27(7.1 \%)$ & $12(7.4 \%)$ & \\
\hline $18.5-25$ & $258(67.7 \%)$ & $116(71.2 \%)$ & \\
\hline$\geq 25$ & $96(25.2 \%)$ & $35(21.5 \%)$ & \\
\hline $\mathrm{HBsAg}$ & & & 0.327 \\
\hline Positive & $328(86.1 \%)$ & $135(82.8 \%)$ & \\
\hline Negative & $53(13.9 \%)$ & $28(17.2 \%)$ & \\
\hline \multicolumn{4}{|l|}{ HBV-DNA (copies/ml) } \\
\hline$<10^{3}$ & $176(46.2 \%)$ & 79 (48.5\%) & 0.645 \\
\hline$\geq 10^{3}$ & $205(53.8 \%)$ & $84(51.5 \%)$ & \\
\hline Liver cirrhosis & & & 0.142 \\
\hline Present & $263(69.0 \%)$ & $102(62.6 \%)$ & \\
\hline Absent & $118(31.0 \%)$ & $61(37.4 \%)$ & \\
\hline Child-Pugh classification & & & 0.204 \\
\hline$A$ & $367(96.3 \%)$ & $161(98.8 \%)$ & \\
\hline B & $14(3.7 \%)$ & $2(1.2 \%)$ & \\
\hline Previous abdominal surgery & & & 0.705 \\
\hline Present & $61(16.0 \%)$ & $24(14.7 \%)$ & \\
\hline Absent & $320(84.0 \%)$ & 139 (85.3\%) & \\
\hline Comorbidities & & & 0.012 \\
\hline Present & 73 (19.2\%) & 17 (10.4\%) & \\
\hline Absent & $308(80.8 \%)$ & 146 (89.6\%) & \\
\hline AFP, ng/mL & & & 0.091 \\
\hline$<400$ & $233(61.2 \%)$ & 87 (53.4\%) & \\
\hline$\geq 400$ & $148(38.8 \%)$ & 76 (46.6\%) & \\
\hline CEA, ng/mL & & & 0.575 \\
\hline Normal & 297 (78.0\%) & 131 (80.4\%) & \\
\hline Abnormal & $84(22.0 \%)$ & $32(19.6 \%)$ & \\
\hline CA19-9, U/ml & & & 0.588 \\
\hline Normal & $233(61.2 \%)$ & $104(63.8 \%)$ & \\
\hline Abnormal & $148(38.8 \%)$ & 59 (36.2\%) & \\
\hline TBIL, umol/L & $14.0(10.9-17.8)$ & $13.7(11.0-18.4)$ & 0.794 \\
\hline DBIL, umol/L & $5.4(4.1-6.8)$ & $5.3(4.2-6.8)$ & 0.853 \\
\hline ALT, IU/L & $38.0(27.0-56.8)$ & $39.0(25.0-62.0)$ & 0.974 \\
\hline AST, IU/L & $38.0(30.0-58.0)$ & $39.0(30.0-59.0)$ & 0.875 \\
\hline Albumin, g/L & & & 0.909 \\
\hline$<35$ & 27 (7.1\%) & $12(7.4 \%)$ & \\
\hline$\geq 35$ & $354(92.9 \%)$ & $151(92.6 \%)$ & \\
\hline NLR & $2.2(1.7-3.1)$ & $2.3(1.6-3.2)$ & 0.776 \\
\hline$P L R$ & $92.9(65.1-128.3)$ & $84.7(64.0-135.9)$ & 0. \\
\hline
\end{tabular}


Table 1 Clinicopathological factors of 544 patients who underwent radical hepatectomy (Continued)

\begin{tabular}{|c|c|c|c|}
\hline Variables & Training cohort $(n=381)$ & Validation cohort $(n=163)$ & $P$ \\
\hline ASA grade & & & 0.088 \\
\hline$\|$ & $316(82.9 \%)$ & $125(76.7 \%)$ & \\
\hline III & $65(17.1 \%)$ & $38(23.3 \%)$ & \\
\hline Largest tumor size, $\mathrm{cm}$ & $5.0(3.2-7.8)$ & $5.6(3.4-9.0)$ & 0.106 \\
\hline Tumor number & & & 0.965 \\
\hline Solitary & $358(94.0 \%)$ & $153(93.9 \%)$ & \\
\hline Multiple & $23(6.0 \%)$ & $10(6.1 \%)$ & \\
\hline Hepatectomy & & & 0.297 \\
\hline Anatomical & $213(55.9 \%)$ & 99 (60.7\%) & \\
\hline Nonanatomical & $168(44.1 \%)$ & $64(39.3 \%)$ & \\
\hline Hemorrhage, ml & & & 0.498 \\
\hline$<200$ & $133(34.9 \%)$ & $52(31.9 \%)$ & \\
\hline$\geq 200$ & $248(65.1 \%)$ & $111(68.1 \%)$ & \\
\hline Intraoperative transfusion & & & 0.606 \\
\hline Yes & $30(7.9 \%)$ & $15(9.2 \%)$ & \\
\hline No & $351(92.1 \%)$ & $148(90.8 \%)$ & \\
\hline Differentiation & & & 0.927 \\
\hline poor & $169(44.4 \%)$ & $73(44.8 \%)$ & \\
\hline Well-moderate & $212(55.6 \%)$ & $90(55.2 \%)$ & \\
\hline MVI & & & 0.477 \\
\hline Present & $124(32.5 \%)$ & $48(29.4 \%)$ & \\
\hline Absent & $257(67.5 \%)$ & $115(70.6 \%)$ & \\
\hline Capsule & & & 0.789 \\
\hline Incomplete & $215(56.4 \%)$ & $94(57.7 \%)$ & \\
\hline Complete & $166(43.6 \%)$ & 69 (42.3\%) & \\
\hline
\end{tabular}

ASA American Society of Anesthesiologists, BMI Body mass index, AFP a-fetoprotein, ALT Alanine transaminase, AST Aspartate aminotransferase, NLR Neutrophil-tolymphocyte ratio, PLR Platelet lymphocyte ratio, MVI Microvascular invasion

univariate analysis, HBsAg, HBV-DNA, AFP, NLR, PLR, largest tumor size, hemorrhage, intraoperative transfusion, differentiation, MVI, capsule and Rad-score were potential risk factors for OS. However, the results of the multivariate analysis suggested that only AFP (HR 1.566; CI 1.101-2.226; $p=0.013$ ), PLR (HR 1.004; CI 1.001$1.007 ; p=0.010$ ), largest tumor size (HR 1.084; CI $1.027-1.145 ; p=0.003$ ), MVI (HR 2.509; CI 1.751-3.594; $p<0.001$ ) and Rad-score (HR 1.398; CI 1.188-1.646; $p<$ 0.001 ) were independently associated with an unfavorable postoperative survival. The radiomics nomogram was constructed with the 5 independent risk predictors identified above to predict the personalized survival status of the patients, while the clinicopathologic nomogram incorporated only the independent clinicopathological risk factors. The C-index of the clinicopathologic nomogram was $0.726(95 \%$ CI $0.705-0.748)$ in the training cohort and 0.720 (95\% CI $0.686-0.755)$ in the validation cohort. The radiomics nomogram yielded a $\mathrm{C}$ - index of 0.747 (95\% CI, 0.727-0.768) in the training cohort and 0.777 (95\% CI, 0.748-0.806) in the validation cohort. The radiomics nomogram showed improved discrimination performance when the radiomics signature was integrated into the clinicopathologic nomogram $(P=0.002$ in the training cohort, $p<0.001$ in the validation cohort; Table 4). The radiomics nomogram and the corresponding calibration curve are presented in Fig. 3. The calibration curve demonstrated satisfactory consistency between the nomogram-predicted survival and the actual observed survival in both the training and validation cohorts.

\section{Clinical utility}

The DCA of the radiomics and clinicopathologic nomograms is presented in Fig. 4. The net benefit was calculated by adding the true positives and subtracting the false positives. The straight line represents the assumption that all patients will die, and the horizontal line 

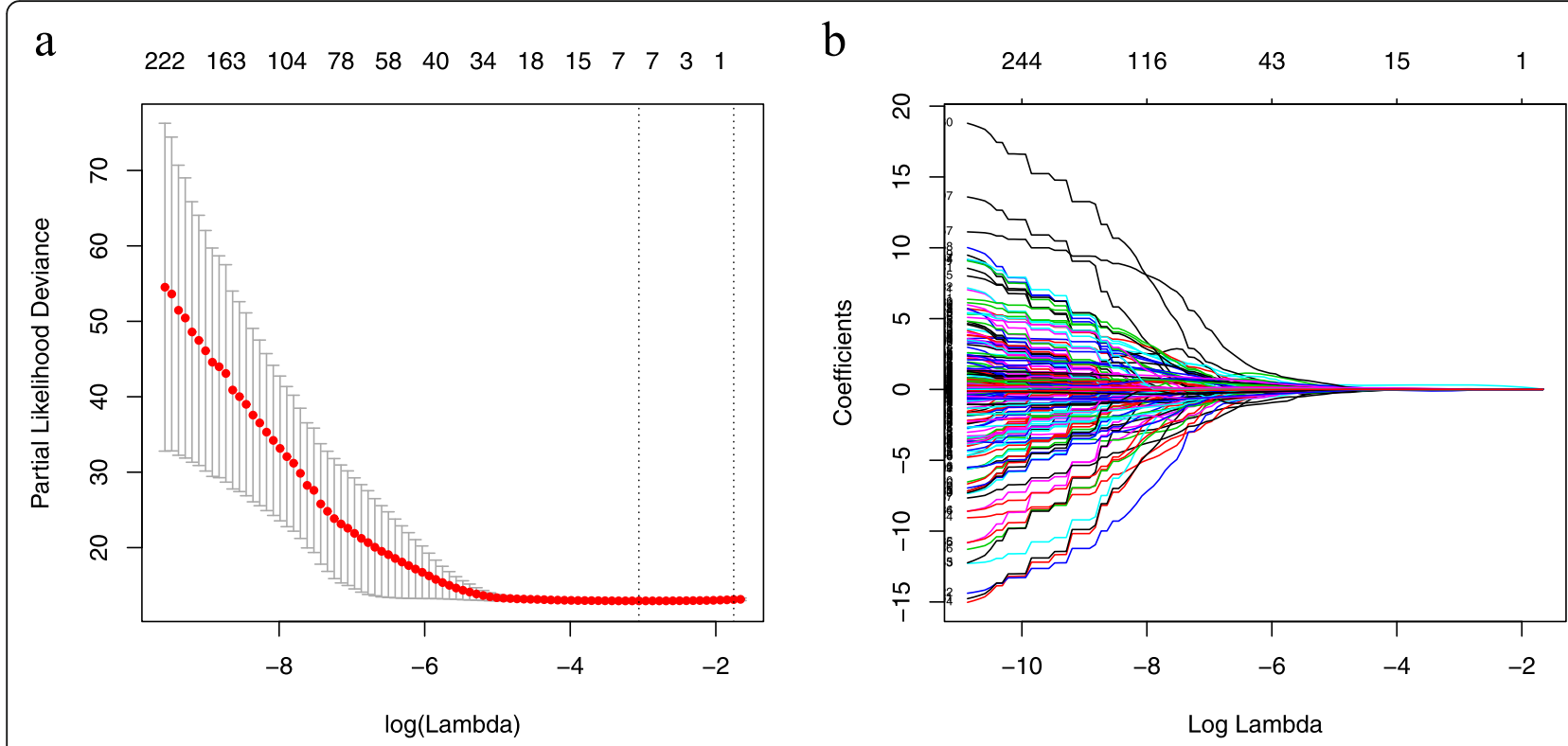

Fig. 1 Radiomics feature selection using the LASSO Cox regression model. a The partial likelihood deviance was plotted versus log (lambda). The $y$-axis indicates the partial likelihood deviance, while the lower $x$-axis indicates the log (lambda) and the upper $x$-axis represents the average number of predictors. Dotted vertical lines were drawn at the optimal values using the minimum criteria and 1 standard error of the minimum criteria. The tuning parameter $(\lambda)$ was selected in the LASSO model via 10-fold cross-validation based on minimum criteria. $\mathbf{b}$ LASSO coefficient profiles of the 270 radiomics features. The coefficients (y-axis) were plotted against log (lambda) and 7 features with nonzero coefficients were selected to build the radiomics signature

represents the assumption that no patients will die. The DCA demonstrated that the nomograms added more net benefit compared with the treat-all strategy or treat-none strategy with a threshold probability of $10 \%$ or greater. Moreover, the radiomics nomogram provided a higher net benefit than the clinicopathologic nomogram in terms of survival prediction in HCC patients.

\section{Discussion}

Surgical resection is the mainstay curative treatment for individuals with HCC, but the prognosis varies from patient to patient. The prediction of survival status in patients with HCC after surgery is important for clinical decision-making. Among the numerous prognostic factors, tumor heterogeneity is one of the most important contributions, which may relate to different natural histories, environmental susceptibility and individual genetic tendencies [36]. Intra-tumoral heterogeneity can reveal tumor growth, metastatic potential and response to treatment and may thus be a potential prognostic predictor of disease outcome [37]. However, previous studies were mainly based on clinicopathological factors [38, 39] and either seldom involved imaging information or

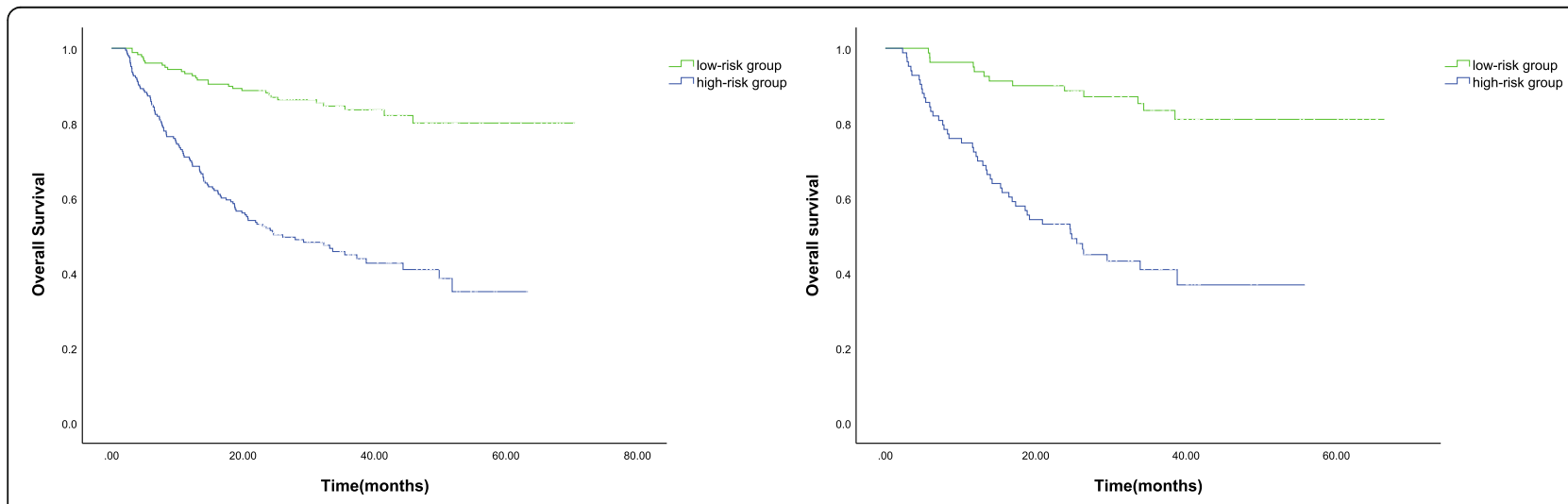

Fig. 2 Kaplan-Meier analyses of overall survival according to the risk groups. a The overall survival of patients in the high- and low-risk groups in the training cohort. $\mathbf{b}$ The overall survival of patients in the high- and low-risk groups in the validation cohort 
Table 2 The clinicopathological data of patients according to the risk-stratified groups in the training cohort

\begin{tabular}{|c|c|c|c|}
\hline Variables & high-risk group $(n=201)$ & low-risk group $(n=180)$ & $P$ \\
\hline Age, years & $51.7 \pm 11.3$ & $50.8 \pm 11.0$ & 0.409 \\
\hline Sex & & & 0.551 \\
\hline Male & $173(86.1 \%)$ & $151(83.9 \%)$ & \\
\hline Female & $28(13.9 \%)$ & $29(16.1 \%)$ & \\
\hline $\mathrm{BMI}, \mathrm{Kg} / \mathrm{m}^{2}$ & & & 0.258 \\
\hline$<18.5$ & $17(8.5 \%)$ & $10(5.5 \%)$ & \\
\hline $18.5-25$ & $129(64.2 \%)$ & $130(72.2 \%)$ & \\
\hline$\geq 25$ & $55(27.4 \%)$ & $40(22.2 \%)$ & \\
\hline $\mathrm{HBsAg}$ & & & 0.367 \\
\hline Positive & $170(84.6 \%)$ & $158(87.8 \%)$ & \\
\hline Negative & $31(15.4 \%)$ & $22(12.2 \%)$ & \\
\hline HBV-DNA (copies/ml) & & & 0.068 \\
\hline$<10^{3}$ & $84(41.8 \%)$ & $92(51.1 \%)$ & \\
\hline$\geq 10^{3}$ & $117(58.2 \%)$ & $88(48.9 \%)$ & \\
\hline Liver cirrhosis & & & 0.086 \\
\hline Present & $131(65.2 \%)$ & $132(73.3 \%)$ & \\
\hline Absent & 70 (34.8\%) & $48(26.7 \%)$ & \\
\hline Child-Pugh classification & & & 0.738 \\
\hline A & $193(96.0 \%)$ & $174(96.7 \%)$ & \\
\hline B & $8(4.0 \%)$ & $6(3.3 \%)$ & \\
\hline Previous abdominal surgery & & & 0.285 \\
\hline Present & $36(17.9 \%)$ & 25 (13.9\%) & \\
\hline Absent & $165(82.1 \%)$ & 155 (86.1\%) & \\
\hline Comorbidities & & & 0.242 \\
\hline Present & $43(21.4 \%)$ & $30(16.7 \%)$ & \\
\hline Absent & $158(78.6 \%)$ & $150(83.3 \%)$ & \\
\hline AFP, ng/mL & & & 0.007 \\
\hline$<400$ & $110(54.7 \%)$ & $123(68.3 \%)$ & \\
\hline$\geq 400$ & $91(45.3 \%)$ & 57 (31.7\%) & \\
\hline CEA, ng/mL & & & 0.543 \\
\hline Normal & 159 (79.1\%) & 138 (76.7\%) & \\
\hline Abnormal & 42 (20.9\%) & $42(23.3 \%)$ & \\
\hline CA19-9, U/ml & & & 0.639 \\
\hline Normal & $121(60.2 \%)$ & $112(62.2 \%)$ & \\
\hline Anormal & 80 (39.8\%) & 68 (37.8\%) & \\
\hline TBIL, umol/L & $14(11.1-18.1)$ & $14.0(10.7-17.3)$ & 0.948 \\
\hline DBIL, umol/L & $5.6(4.2-7.0)$ & $5.4(4.0-6.8)$ & 0.375 \\
\hline$A L T, I U / L$ & $38.0(26.0-56.5)$ & $37.0(27.0-56.5)$ & 0.880 \\
\hline AST, IU/L & $43.0(31.0-72.0)$ & $36.0(28.0-45.8)$ & $<0.001$ \\
\hline Albumin, $\mathrm{g} / \mathrm{L}$ & & & 0.483 \\
\hline$<35$ & $16(8.0 \%)$ & $11(6.1 \%)$ & \\
\hline$\geq 35$ & 185 (92.0\%) & 169 (93.9\%) & \\
\hline NLR & $2.2(1.7-3.1)$ & $2.2(1.7-2.9)$ & 0.113 \\
\hline PLR & $92.9(65.1-128.3)$ & $83.7(62.6-114.3)$ & 0.001 \\
\hline
\end{tabular}


Table 2 The clinicopathological data of patients according to the risk-stratified groups in the training cohort (Continued)

\begin{tabular}{|c|c|c|c|}
\hline Variables & high-risk group $(n=201)$ & low-risk group $(n=180)$ & $P$ \\
\hline ASA grade & & & 0.199 \\
\hline$\|$ & $162(80.6 \%)$ & $154(85.6 \%)$ & \\
\hline III & 39 (19.4\%) & $26(14.4 \%)$ & \\
\hline Largest tumor size, $\mathrm{cm}$ & $7.0(5.0-10.0)$ & $3.7(2.5-5.0)$ & $<0.001$ \\
\hline Tumor number & & & 0.709 \\
\hline Solitary & $188(93.5 \%)$ & $170(94.4 \%)$ & \\
\hline Multiple & $13(6.5 \%)$ & $10(5.6 \%)$ & \\
\hline Hepatectomy & & & 0.245 \\
\hline Anatomical & $118(58.7 \%)$ & 95 (52.8\%) & \\
\hline Nonanatomical & $83(41.3 \%)$ & $85(47.2 \%)$ & \\
\hline Hemorrhage, ml & & & $<0.001$ \\
\hline$<200$ & $53(26.4 \%)$ & $80(44.4 \%)$ & \\
\hline$\geq 200$ & $148(73.6 \%)$ & $100(55.6 \%)$ & \\
\hline Intraoperative transfusion & & & 0.019 \\
\hline Yes & $22(10.9 \%)$ & $8(4.4 \%)$ & \\
\hline No & $179(89.1 \%)$ & $172(95.6 \%)$ & \\
\hline Differentiation & & & 0.068 \\
\hline poor & $98(48.8 \%)$ & 71 (39.4\%) & \\
\hline Well-moderate & $103(51.2 \%)$ & 109 (60.6\%) & \\
\hline $\mathrm{MVI}$ & & & $<0.001$ \\
\hline Present & $87(43.3 \%)$ & $37(20.6 \%)$ & \\
\hline Absent & $114(56.7 \%)$ & $143(79.4 \%)$ & \\
\hline Capsule & & & $<0.001$ \\
\hline Incomplete & $137(68.2 \%)$ & $78(43.3 \%)$ & \\
\hline Complete & $64(31.8 \%)$ & $102(56.7 \%)$ & \\
\hline Rad-score & $-0.1(-0.3 \sim 0.5)$ & $-0.8(-1.0 \sim-0.7)$ & $<0.001$ \\
\hline
\end{tabular}

$\overline{A S A}$ American Society of Anesthesiologists, BMI Body mass index, AFP a-fetoprotein, ALT Alanine transaminase, AST Aspartate aminotransferase, NLR Neutrophil-tolymphocyte ratio, PLR Platelet lymphocyte ratio, MVI Microvascular invasion, Rad-score Radiomics score

only considered a small number of subjective imaging parameters [40, 41]. Moreover, a large amount of tumor-related information that can be extracted from images is ignored. Radiomics can capture the potential heterogeneity of lesions using a large number of quantitative imaging features, which may be a valuable supplement to the existing predictors.

Medical imaging plays an important role in preoperative diagnosis, choice of therapy, therapeutic effect evaluation and disease surveillance. However, the interpretation of medical imaging is often based on physicians' personal expertise and experience, which are subjective and qualitative. Radiomics can be used to analyze the texture parameters extracted by a computer and can allow the quantitative assessment of the pixel differences in images to provide more comprehensive information about tumors that may not be detected by the human eye. In addition, the temporal and spatial heterogeneity of the tumor can be evaluated by whole tumor analysis instead of in limited biopsy samples [42]. Medical imaging analysis can reveal the tumor biological processes and microenvironment characteristics and may assist in therapeutic decision-making. However, few studies have focused on the prognostic prediction in patients with HCC. Therefore, this study aimed to develop a radiomics signature to predict the prognosis of patients with HCC after surgical resection based on selected radiomics features. Moreover, a nomogram was constructed based on the independent risk factors, which allows for more precise prognostication, better clinical management and more appropriate adjuvant therapy. This study introduced a noninvasive, low cost and reproducible method to predict the outcomes in patients with resectable HCC, which is of great significance for personalized medicine. 
Table 3 Univariate and multivariate Cox regression analyses for patients in the training cohort

\begin{tabular}{|c|c|c|c|c|}
\hline \multirow[t]{2}{*}{ Variables } & \multicolumn{2}{|l|}{ Univariate analysis } & \multicolumn{2}{|c|}{ Multivariate analysis } \\
\hline & HR $(95 \% \mathrm{Cl})$ & $p$ & HR $(95 \% \mathrm{Cl})$ & $p$ \\
\hline Age, years & $0.986(0.971-1.000)$ & 0.052 & & \\
\hline Sex, male vs. female & $1.675(0.964-2.911)$ & 0.067 & & \\
\hline \multicolumn{5}{|l|}{$\mathrm{BMI}, \mathrm{Kg} / \mathrm{m}^{2}$} \\
\hline$<18.5 v s .18 .5-25$ & $0.944(0.455-1.957)$ & 0.876 & & \\
\hline $25 v s .18 .5-25$ & $1.171(0.539-2.541)$ & 0.691 & & \\
\hline HBsAg, Positive vs. Negative & $1.950(1.079-3.523)$ & 0.027 & & \\
\hline HBV-DNA, copies $/ \mathrm{ml},<10^{3}$ vs. $\geq 10^{3}$ & $1.740(1.223-2.476)$ & 0.002 & & \\
\hline Liver cirrhosis, Present vs. Absent & $0.826(0.583-1.171)$ & 0.283 & & \\
\hline Child-Pugh classification, B vs. A & $1.684(0.825-3.438)$ & 0.152 & & \\
\hline Previous abdominal surgery, Present vs. Absent & $0.893(0.562-1.420)$ & 0.632 & & \\
\hline Comorbidities, Present vs. Absent & $1.016(0.668-1.546)$ & 0.941 & & \\
\hline AFP, ng/mL, $\geq 400 v s .<400$ & $1.931(1.388-2.688)$ & $<0.001$ & $1.566(1.101-2.226)$ & 0.013 \\
\hline CEA, ng/mL, Normal vs. Abnormal & $0.656(0.419-1.027)$ & 0.065 & & \\
\hline CA19-9, U/ml, Normal vs. Abnormal & $1.131(0.807-1.585)$ & 0.474 & & \\
\hline TBIL, umol/L & $1.000(0.988-1.013)$ & 0.940 & & \\
\hline DBIL, umol/L & $0.999(0.982-1.016)$ & 0.901 & & \\
\hline $\mathrm{ALT}, \mathrm{IU} / \mathrm{L}$ & $1.000(0.999-1.002)$ & 0.823 & & \\
\hline AST, IU/L & $1.000(0.999-1.002)$ & 0.423 & & \\
\hline Albumin, $g / L,<35 v s . \geq 35$ & $1.322(0.732-2.390)$ & 0.355 & & \\
\hline NLR & $1.085(1.030-1.143)$ & 0.002 & & \\
\hline PLR & $1.006(1.004-1.009)$ & $<0.001$ & $1.004(1.001-1.007)$ & 0.010 \\
\hline ASA grade, III vs. II & $1.362(0.910-2.039)$ & 0.133 & & \\
\hline Largest tumor size, $\mathrm{cm}$ & $1.193(1.145-1.244)$ & $<0.001$ & $1.084(1.027-1.145)$ & 0.003 \\
\hline Tumor number, Solitary vs. Multiple & $1.314(0.710-2.432)$ & 0.384 & & \\
\hline Hepatectomy, Anatomical vs. Nonanatomical & $0.755(0.538-1.060)$ & 0.105 & & \\
\hline Hemorrhage, $\mathrm{ml}, \geq 200 \mathrm{vs} .<200$ & $1.927(1.310-2.836)$ & 0.001 & & \\
\hline Intraoperative transfusion, Yes vs. No & $1.860(1.104-3.132)$ & 0.020 & & \\
\hline Differentiation, poor vs. Well-moderate & $1.598(1.148-2.225)$ & 0.006 & & \\
\hline MVI, Present vs. Absent & $3.524(2.524-4.921)$ & $<0.001$ & $2.509(1.751-3.594)$ & $<0.001$ \\
\hline Capsule, Incomplete vs. Complete & $1.891(1.324-2.702)$ & $<0.001$ & & \\
\hline Rad-score & $1.493(1.324-1.684)$ & $<0.001$ & $1.398(1.188-1.646)$ & $<0.001$ \\
\hline
\end{tabular}

ASA American Society of Anesthesiologists, BMI Body mass index, AFP a-fetoprotein, ALT Alanine transaminase, AST Aspartate aminotransferase, NLR Neutrophil-tolymphocyte ratio, PLR Platelet lymphocyte ratio, MVI Microvascular invasion, Rad-score Radiomics score

Table 4 Performance of the radiomics and clinicopathologic nomogram for Prediction of OS

\begin{tabular}{|c|c|c|c|c|}
\hline \multirow[t]{2}{*}{ Nomogram } & \multicolumn{2}{|l|}{ The training cohort } & \multicolumn{2}{|c|}{ The validation cohort } \\
\hline & C-index & $P$-value & C-index & $P$-value \\
\hline Radiomics & $0.747(0.727-0.768)$ & 0.002 & $0.777(0.748-0.806)$ & $<0.001$ \\
\hline Clinicopathological & $0.726(0.705-0.748)$ & & $0.720(0.686-0.755)$ & \\
\hline
\end{tabular}




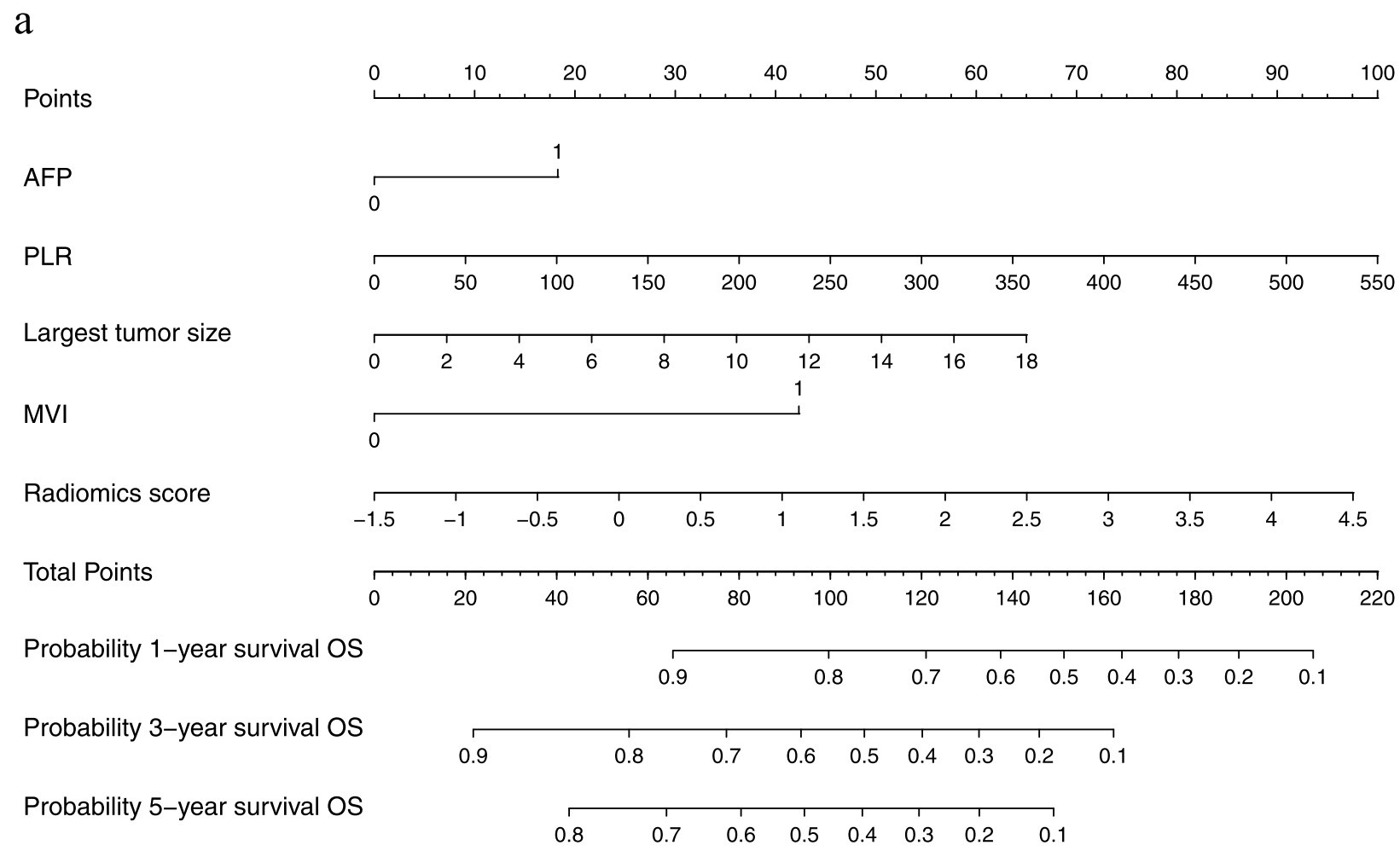

b

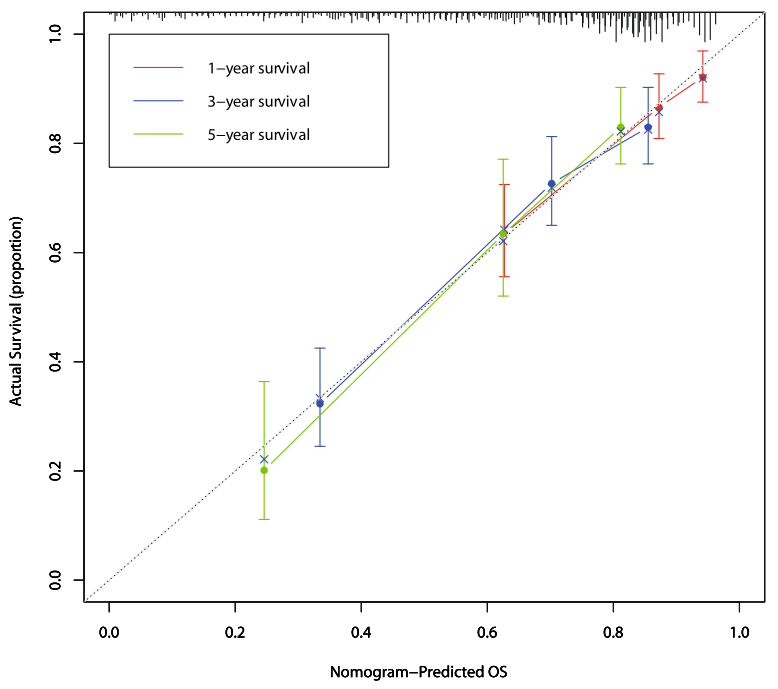

C

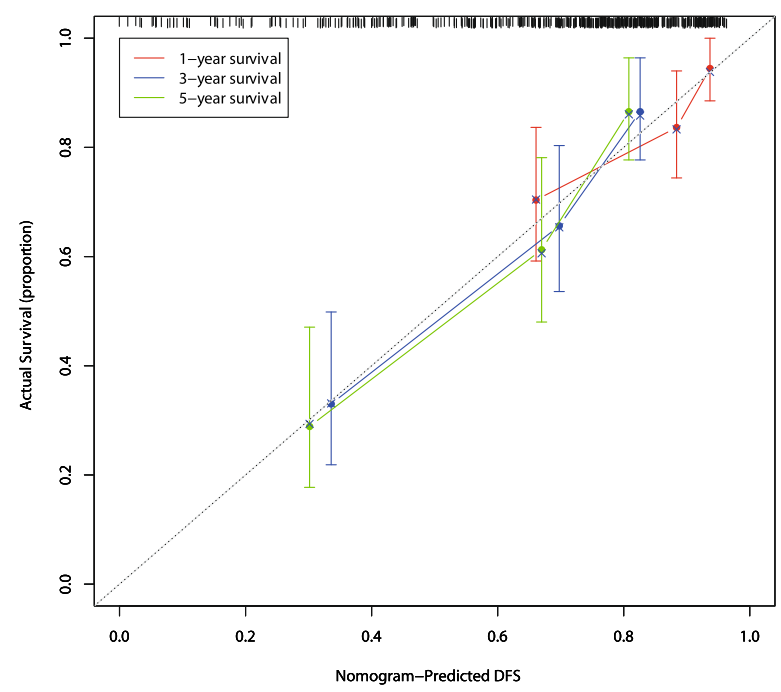

Fig. 3 The radiomics nomogram for the prediction of survival status (a). The calibration curves of the radiomics nomogram in the training cohort (b) and the validation cohort (c)

In our study, 5 optimal features were selected from 270 radiomics features of the portal venous phase via the LASSO method to build a radiomics signature, after which the patients were divided into the high-risk and low-risk groups according to the Rad-score threshold. The results indicate that patients with higher Rad-scores were more likely to have a worse OS than those with lower Rad-scores. In the multivariate analysis, the radiomics signature was further demonstrated to be an independent predictor of OS. This study provides a method for prognosis-related high-dimensional data selection. LASSO is a penalized regression approach that selects 


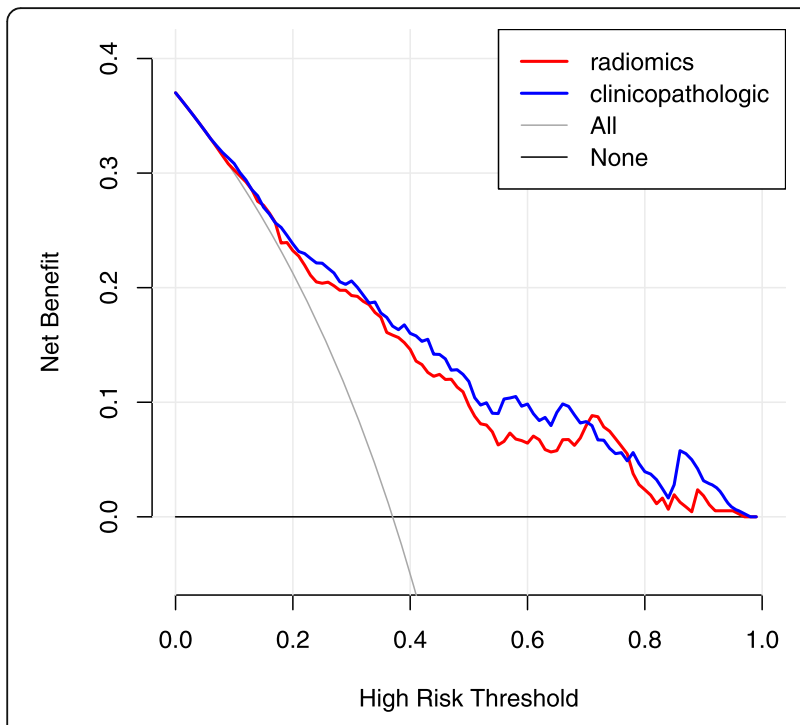

Fig. 4 Decision curve analysis of the radiomics and clinicopathologic nomogram in the entire cohort $(n=544)$. The $y$-axis represents the net benefit, and the $x$-axis represents the threshold probability. The black line represents the assumption that no patients exhibited long-term overall survival (OS). The grey line represents the assumption that all patients exhibited long-term OS. The decision curves indicated that the radiomics nomogram (red line) showed better clinical utility than the clinicopathologic nomogram (blue line)

covariates with non-zero coefficients among numerous covariates to avoid overfitting, thus improving the prediction efficiency [43]. Those radiomic features provided a quantitative description of the position, intensity and inter-relationship of the pixels $[12,44]$ to reveal tumor phenotypic differences and to evaluate the intra-tumor heterogeneity, which is related to tumor proliferation, hypoxia, angiogenesis and necrosis. Increased homogeneity in colorectal cancer was related to a poor prognosis, while increased heterogeneity in oesophageal cancer and gastric cancer was associated with a poor prognosis [4548]. Entropy and uniformity are common texture parameters, and higher entropy and lower uniformity reflected increased tumor heterogeneity [49]. However, a large number of texture parameters related to tumor aggressiveness have not been well studied.

The present study showed that AFP, PLR, largest tumor size, MVI and the radiomics signature were independent risk factors for OS. In agreement with the previous study, these clinicopathologic factors are known to be effective predictors of the clinical outcome [50-53].It has also been noted that inflammatory markers were related to HCC aggressiveness, and in our study, PLR was included in the final model [54]. However, the tumor number and tumor differentiation were not associated with survival status in our study. The possible reasons for this are the limited cases of multifocal lesions and the short follow-up time in the present study. Furthermore, in the subgroup analysis, patients in the high-risk group tended to show higher AFP, higher AST, higher PLR, larger tumor size, more hemorrhage, more intraoperative transfusion, the presence of MVI and an incomplete tumor capsule, which are demonstrated prognostic indicators of HCC $[38,39,55,56]$, indicating the potential association between the radiomics signature and clinicopathologic factors. Therefore, texture parameters are linked to clinicopathologic factors, which could assist clinicians in prognostic evaluation.

We established a combined nomogram that incorporates clinicopathologic factors and a radiomics signature for prognostic prediction at the individual level. The results indicated that the radiomics nomogram showed improved predictive accuracy over the clinicopathologic nomogram, which indicates that the radiomics signature can provide additional prognostic and biologic information; this is consistent with previous studies. Meng et al. [57] performed a study in 108 consecutive patients with locally advanced rectal cancer, and the results implied that the combined model $(C$-index $=0.788)$ exhibited improved predictive ability of the 3-year disease-free survival compared with the radiomic $(C$-index $=0.767)$ and clinicoradiologic models $(\mathrm{C}$-index $=0.644)$. Li et al. [58] explored the prognostic value of radiomics in $181 \mathrm{pa}-$ tients with gastric cancer following curative resection and revealed that the radiomics nomogram (C-index $=$ 0.82) showed better predictive ability than the clinical nomogram $(C$-index $=0.71)$ and the radiomics signature $(C$-index $=0.74)$. Based on the results of our study, aggressive precautions can be taken in patients with predicted poor prognoses, which would facilitate the effective therapeutic management of these patients and reduce the risk of recurrence.

The limitations of this study are as follows: (1) this study is a retrospective single-center study with a small sample size, and the results of the study are limited. In addition, the model was only verified internally and lacked external validation. (2) Contrast-enhanced CT was used in our study, whereas contrast-enhanced MRI can capture more microstructural characteristics of the tumors and may provide more comprehensive information pertaining to tumor heterogeneity [59]. (3) In our study, only the largest cross-section of a lesion in the portal phase was analyzed, while whole tumor analysis in both the arterial and portal phases may improve the efficiency of survival prediction in individuals with HCC. (4) Although the ROIs were derived from manual segmentation by two radiologists, their subjective bias could not be completely eliminated. Therefore, further studies are warranted to confirm our results. 


\section{Conclusion}

In conclusion, the radiomics signature provided a quantitative method for the assessment of survival status in patients with HCC after hepatectomy. The patients with high Rad-scores may experience a higher risk of recurrence and metastasis. Moreover, the radiomics nomogram that integrates clinicopathological factors and the radiomics signature may serve as an effective tool to guide the individualized management and tailored follow-up of HCC patients. In the future, multicenter prospective studies are needed to further investigate the potential value of the radiomics signature in clinical practice.

\section{Supplementary Information}

The online version contains supplementary material available at https://doi. org/10.1186/s40644-020-00360-9.

Additional file 1: Table S1. Texture features used in the study.

\begin{abstract}
Abbreviations
HCC: Hepatocellular carcinoma; TA: Texture analysis; CT: Computed tomography; LASSO: Least absolute shrinkage and selection operator; Radscore: Radiomics score; ROC: Receiver operating characteristic; OS: Overall survival; C-index: Concordance index; DCA: Decision curve analysis; AFP: afetoprotein; PLR: Platelet-to-lymphocyte ratio; MVI: Microvascular invasion; BCLC: Barcelona Clinic Liver Cancer; TACE: Transcatheter arterial chemoembolization; HBV-DNA: Hepatitis B virus deoxyribonucleic acid; DICOM: Digital Imaging and Communications in Medicine; ROls: Regions of interest; ASA: American Society of Anesthesiologists; BMl: Body mass index; ALT: Alanine transaminase; AST: Aspartate aminotransferase; NLR: Neutrophilto-lymphocyte ratio
\end{abstract}

\section{Acknowledgements}

Not applicable.

\section{Authors' contributions}

(I) Conception and design: Weixia Chen, Yonggang Wei, Bo Li, Lu Zheng; (II) Administrative support: None; (III) Provision of study materials or patients: All authors; (IV) Collection and assembly of data: Qinqin Liu, Jing Li, Fei Liu, Weilin Yang, Jingjing Ding; (V) Data analysis and interpretation: Qinqin Liu, Jing Li, Fei Liu, Weilin Yang; (VI) Manuscript writing: All authors; (VII) Final approval of manuscript: All authors.

\section{Funding}

This study was supported by the Medical Research Project jointly funded by Chongqing Science and Technology Commission and Chongqing Health Commission (2019ZDXM046), the Technological Innovation and Application Demonstration Special Project of Chongqing (cstc2018jscx-mszdX0012), and the Scientific and Technological Innovation Special Project of Army Medical University (2019XLC2006).

\section{Availability of data and materials}

The datasets generated during and/or analyzed during the current study are available from the corresponding author on reasonable request.

\section{Ethics approval and consent to participate}

The study was approved by the Committee of Ethics in West China Hospital of Sichuan University. Informed consent was obtained from each individual for the study.

\section{Consent for publication}

Not applicable.

\section{Competing interests}

The authors declare that they have no competing interests.

\section{Author details}

${ }^{1}$ Department of Liver Surgery, Center of Liver Transplantation, West China Hospital, Sichuan University, 37 Guo Xue Road, Chengdu 610041, Sichuan Province, China. ${ }^{2}$ Department of Hepatobiliary Surgery, the Second Affiliated Hospital of Army Medical University, No. 183 Xinqiao High Street, Shapingba District, Chongqing 400037, China. ${ }^{3}$ The First Affiliated Hospital of Jinan University, Guangzhou, China. ${ }^{4}$ Department of Radiology, West China Hospital, Sichuan University, Chengdu, China. ${ }^{5}$ Department of Gastrointestinal Surgery, West China Hospital, Sichuan University, Chengdu, China.

Received: 15 June 2020 Accepted: 28 October 2020

Published online: 16 November 2020

\section{References}

1. El-Serag HB, Rudolph KL. Hepatocellular carcinoma: epidemiology and molecular carcinogenesis. Gastroenterology. 2007;132(7):2557-76.

2. Jemal A, Bray F, Center MM, Ferlay J, Ward E, Forman D. Global cancer statistics. CA Cancer J Clin. 2011;61(2):69-90.

3. European Association For The Study Of The Liver; European Organisation For Research And Treatment Of Cancer. EASL-EORTC clinical practice guidelines: management of hepatocellular carcinoma. J Hepatol. 2012;56(4): 908-43.

4. Earl TM, Chapman WC. Hepatocellular carcinoma: resection versus transplantation. Semin Liver Dis. 2013;33(3):282-92.

5. Poon RT, Fan ST, Lo CM, Liu CL, Wong J. Long-term survival and pattern of recurrence after resection of small hepatocellular carcinoma in patients with preserved liver function: implications for a strategy of salvage transplantation. Ann Surg. 2002;235(3):373-82.

6. Shah SA, Cleary SP, Wei AC, et al. Recurrence after liver resection for hepatocellular carcinoma: risk factors, treatment, and outcomes. Surgery. 2007;141(3):330-9

7. Lacaze L, Scotte M. Surgical treatment of intra hepatic recurrence of hepatocellular carcinoma. World J Hepatol. 2015;7(13):1755-60.

8. Vitale A, Burra P, Frigo AC, et al. Survival benefit of liver resection for patients with hepatocellular carcinoma across different Barcelona clinic liver cancer stages: a multicentre study. J Hepatol. 2015;62(3):617-24.

9. Bolondi L, Burroughs A, Dufour JF, et al. Heterogeneity of patients with intermediate (BCLC B) hepatocellular carcinoma: proposal for a subclassification to facilitate treatment decisions. Semin Liver Dis. 2012;32(4): 348-59.

10. Huitzil-Melendez FD, Capanu M, O'Reilly EM, et al. Advanced hepatocellular carcinoma: which staging systems best predict prognosis? J Clin Oncol. 2010;28(17):2889-95

11. Roberts $L R$, Sirlin $C B$, Zaiem F, et al. Imaging for the diagnosis of hepatocellular carcinoma: a systematic review and meta-analysis. Hepatology (Baltimore, Md). 2018;67(1):401-21.

12. Castellano G, Bonilha L, Li LM, Cendes F. Texture analysis of medical images. Clin Radiol. 2004;59(12):1061-9.

13. Lubner MG, Smith AD, Sandrasegaran K, Sahani DV, Pickhardt PJ. CT texture analysis: definitions, applications, biologic correlates, and challenges. Radiographics. 2017;37(5):1483-503.

14. Gillies RJ, Kinahan PE, Hricak H. Radiomics: images are more than pictures, they are data. Radiology. 2016;278(2):563-77.

15. Miles KA, Ganeshan B, Griffiths MR, Young RC, Chatwin CR. Colorectal cancer: texture analysis of portal phase hepatic $C T$ images as a potential marker of survival. Radiology. 2009;250(2):444-52.

16. Ba-Ssalamah A, Muin D, Schernthaner $R$, et al. Texture-based classification of different gastric tumors at contrast-enhanced CT. Eur J Radiol. 2013;82(10): e537-43.

17. Huang YQ, Liang $\mathrm{CH}, \mathrm{He} \mathrm{L}$, et al. Development and validation of a Radiomics Nomogram for preoperative prediction of lymph node metastasis in colorectal cancer. J Clin Oncol. 2016;34(18):2157-64.

18. Wu S, Zheng J, Li Y, et al. A Radiomics Nomogram for the preoperative prediction of lymph node metastasis in bladder cancer. Clin Cancer Res. 2017;23(22):6904-11.

19. Ahn SJ, Kim JH, Park SJ, Han JK. Prediction of the therapeutic response after FOLFOX and FOLFIRI treatment for patients with liver metastasis from 
colorectal cancer using computerized CT texture analysis. Eur J Radiol. 2016; 85(10):1867-74.

20. Peng J, Zhang J, Zhang Q, Xu Y, Zhou J, Liu L. A radiomics nomogram for preoperative prediction of microvascular invasion risk in hepatitis B virusrelated hepatocellular carcinoma. Diagn Interv Radiol. 2018;24(3):121-7.

21. Zhou Y, He L, Huang Y, et al. CT-based radiomics signature: a potential biomarker for preoperative prediction of early recurrence in hepatocellular carcinoma. Abdom Radiol (NY). 2017;42(6):1695-704.

22. Pinker KA-O, Shitano F, Sala E, et al. Background, current role, and potential applications of radiogenomics. J Magn Reson Imaging. 2018;47(3):604.

23. Segal $\mathrm{E}$, Sirlin $\mathrm{CB}$, Ooi C, et al. Decoding global gene expression programs in liver cancer by noninvasive imaging. Nat Biotechnol. 2007;25(6):675-80.

24. Banerjee S, Wang DS, Kim HJ, et al. A computed tomography radiogenomic biomarker predicts microvascular invasion and clinical outcomes in hepatocellular carcinoma. Hepatology. 2015;62(3):792-800.

25. Aerts HJ, Velazquez ER, Leijenaar RT, et al. Decoding tumour phenotype by noninvasive imaging using a quantitative radiomics approach. Nat Commun. 2014:5:4006.

26. Rutman AM, Kuo MD. Radiogenomics: creating a link between molecular diagnostics and diagnostic imaging. Eur J Radiol. 2009;70(2):232-41.

27. Panth KM, Leijenaar RT, Carvalho $S$, et al. Is there a causal relationship between genetic changes and radiomics-based image features? An in vivo preclinical experiment with doxycycline inducible GADD34 tumor cells. Radiother Oncol. 2015;116(3):462-6.

28. Lambin P, Rios-Velazquez E, Leijenaar $R$, et al. Radiomics: extracting more information from medical images using advanced feature analysis. Eur J Cancer (Oxford, England : 1990). 2012;48(4):441-6.

29. Choi JY, Fau LJ, Sirlin CB, Sirlin CB. CT and MR imaging diagnosis and staging of hepatocellular carcinoma: part I. development, growth, and spread: key pathologic and imaging aspects. Radiology. 2014;272(3):635.

30. Cai W, He B, Hu M, et al. A radiomics-based nomogram for the preoperative prediction of posthepatectomy liver failure in patients with hepatocellular carcinoma. Surg Oncol. 2019;28:78-85.

31. Ma X, Wei J, Gu D, et al. Preoperative radiomics nomogram for microvascular invasion prediction in hepatocellular carcinoma using contrast-enhanced CT. Eur Radiol. 2019;29(7):3595-605.

32. Szczypiński PM, Strzelecki M, Materka A, Klepaczko A. MaZda--a software package for image texture analysis. Comput Methods Prog Biomed. 2009; 94(1):66-76

33. Sauerbrei W, Royston $\mathrm{P}$, Binder $\mathrm{H}$. Selection of important variables and determination of functional form for continuous predictors in multivariable model building. Stat Med. 2007:26(30):5512-28.

34. Vasquez MM, Hu C, Roe DJ, Chen Z, Halonen M, Guerra S. Least absolute shrinkage and selection operator type methods for the identification of serum biomarkers of overweight and obesity: simulation and application. BMC Med Res Methodol. 2016;16(1):154.

35. Vickers AJ, Elkin EB. Decision curve analysis: a novel method for evaluating prediction models. Med Dec Making. 2006;26(6):565-74.

36. Li L, Wang H. Heterogeneity of liver cancer and personalized therapy. Cancer Lett. 2016:379(2):191-7.

37. Hlady RA, Robertson KD. Genetic and epigenetic heterogeneity in Normal liver homeostasis and its implications for liver disease and hepatocellular cancer. Semin Liver Dis. 2018:38(1):41-50.

38. Wan G, Gao F, Chen J, et al. Nomogram prediction of individual prognosis of patients with hepatocellular carcinoma. BMC Cancer. 2017;17(1):91.

39. Zhang $W$, Tan $Y$, Jiang $L$, et al. Prognostic nomogram for patients with nonB non-C hepatocellular carcinoma after curative liver resection. Int I Surg. 2017:44:160-5

40. Han JH, Kim DG, Na GH, et al. Evaluation of prognostic factors on recurrence after curative resections for hepatocellular carcinoma. World J Gastroenterol. 2014;20(45):17132-40.

41. Hayano K, Yoshida H, Zhu AX, Sahani DV. Fractal analysis of contrastenhanced CT images to predict survival of patients with hepatocellular carcinoma treated with sunitinib. Dig Dis Sci. 2014;59(8):1996-2003.

42. Limkin EJ, Sun R, Dercle L, et al. Promises and challenges for the implementation of computational medical imaging (radiomics) in oncology. Ann Oncol. 2017;28(6):1191-206.

43. Kim SM, Kim Y, Jeong K, Jeong H, Kim J. Logistic LASSO regression for the diagnosis of breast cancer using clinical demographic data and the BI-RADS lexicon for ultrasonography. Ultrasonography. 2018;37(1):36-42.
44. Chicklore S, Goh V, Siddique M, Roy A, Marsden PK, Cook GJ. Quantifying tumour heterogeneity in 18F-FDG PET/CT imaging by texture analysis. Eur J Nucl Med Mol Imaging. 2013;40(1):133-40.

45. Ganeshan B, Skogen K, Pressney I, Coutroubis D, Miles K. Tumour heterogeneity in oesophageal cancer assessed by $C T$ texture analysis: preliminary evidence of an association with tumour metabolism, stage, and survival. Clin Radiol. 2012;67(2):157-64.

46. Ng F, Ganeshan B, Kozarski R, Miles KA, Goh V. Assessment of primary colorectal cancer heterogeneity by using whole-tumor texture analysis: contrast-enhanced $C T$ texture as a biomarker of 5-year survival. Radiology. 2013;266(1):177-84.

47. Yip C, Landau D, Kozarski R, et al. Primary esophageal cancer: heterogeneity as potential prognostic biomarker in patients treated with definitive chemotherapy and radiation therapy. Radiology. 2014;270(1):141-8.

48. Giganti F, Antunes S, Salerno A, et al. Gastric cancer: texture analysis from multidetector computed tomography as a potential preoperative prognostic biomarker. Eur Radiol. 2017;27(5):1831-9.

49. Ganeshan B, Miles KA, Young RC, Chatwin CR. Hepatic entropy and uniformity: additional parameters that can potentially increase the effectiveness of contrast enhancement during abdominal CT. Clin Radiol. 2007;62(8):761-8.

50. Jing YY, Liu WT, Guo SW, et al. Hepatitis B virus (HBV) receptors: deficiency in tumor results in scant HBV infection and overexpression in peritumor leads to higher recurrence risk. Oncotarget. 2015;6(40):42952-62.

51. Rodriguez-Peralvarez M, Luong TV, Andreana L, Meyer T, Dhillon AP, Burroughs AK. A systematic review of microvascular invasion in hepatocellular carcinoma: diagnostic and prognostic variability. Ann Surg Oncol. 2013;20(1):325-39.

52. Kang SH, Kim DY, Jeon SM, et al. Clinical characteristics and prognosis of hepatocellular carcinoma with different sets of serum AFP and PIVKA-II levels. Eur J Gastroenterol Hepatol. 2012;24(7):849-56.

53. Chang SK, Hlaing WW, Yu RQ, Lee TW, Ganpathi IS, Madhavan KK. Value of alpha-foetoprotein for screening of recurrence in hepatocellular carcinoma post resection. Singap Med J. 2012;53(1):32-5.

54. Wang D, Bai N, Hu X, et al. Preoperative inflammatory markers of NLR and PLR as indicators of poor prognosis in resectable HCC. PeerJ. 2019;7:e7132.

55. Li Z, Zhao $X$, Jiang $P$, et al. HBV is a risk factor for poor patient prognosis after curative resection of hepatocellular carcinoma: a retrospective casecontrol study. Medicine (Baltimore). 2016;95(31):e4224.

56. Gao F, Li X, Geng M, et al. Pretreatment neutrophil-lymphocyte ratio: an independent predictor of survival in patients with hepatocellular carcinoma. Medicine (Baltimore). 2015;94(11):e639.

57. Meng $Y$, Zhang $Y$, Dong $D$, et al. Novel radiomic signature as a prognostic biomarker for locally advanced rectal cancer. J Magn Reson Imaging. 2018

58. Li W, Zhang L, Tian C, et al. Prognostic value of computed tomography radiomics features in patients with gastric cancer following curative resection. Eur Radiol. 2018;29(6):3079.

59. Kitao A, Matsui O, Yoneda N, et al. Hypervascular hepatocellular carcinoma: correlation between biologic features and signal intensity on gadoxetic acid-enhanced MR images. Radiology. 2012;265(3):780-9.

\section{Publisher's Note}

Springer Nature remains neutral with regard to jurisdictional claims in published maps and institutional affiliations.

Ready to submit your research? Choose BMC and benefit from:

- fast, convenient online submission

- thorough peer review by experienced researchers in your field

- rapid publication on acceptance

- support for research data, including large and complex data types

- gold Open Access which fosters wider collaboration and increased citations

- maximum visibility for your research: over $100 \mathrm{M}$ website views per year

At BMC, research is always in progress.

Learn more biomedcentral.com/submissions 